\title{
Per un Diverso Decameron
}

\author{
Renzo BragantinI*
}

ABSTRACT: Che il Decameron sia stato largamente influenzato dalla Commedia dantesca (e anche, in parte, dal Canzoniere petrarchesco) è risaputo. Ma generalmente tale influenza è considerata solo alla stregua di una venerazione di Boccaccio per Dante, senza che ci si sia domandato se Boccaccio anche non intervenga a ridiscutere, in funzione della propria poetica, alcuni assunti basilari del poema dantesco. Il saggio si propone appunto tale compito, tentando di dimostrare come Boccaccio, intervenendo su tali assunti, ne interroghi i principi di fondo, primo tra tutti quello dello statuto di verità, a ogni momento rilanciato da Dante e proposto al proprio lettore, e sottoposto da Boccaccio a un'attenta, e talora critica, verifica. Ciò avviene soprattutto in alcuni brani del Decameron in cui Boccaccio parla direttamente, senza la mediazione dei propri narratori (a esempio, nell'Introduzione alla IV giornata); ma è anche individuabile nelle premesse e conclusioni dei narratori ai propri racconti. In esse l'inattingibilità della verità, e allo stesso tempo la necessità di interpretare la realtà, sottoposta a un incessante scrutinio, si propone come un modello non opposto, ma certo radicalmente e problematicamente altro da quanto suggerito dall'esperienza dantesca.

PAROLE CHIAVE: Decameron; Giovanni Boccaccio; Dante Alighieri; Francesco Petrarca; modelli narrativi; verità; interpretazione. 
RESUMO: Que o Decameron tenha sido fortemente influenciado pela Comédia dantesca (e parcialmente também pelo Cancioneiro de Petrarca) é fato notório. Em geral, porém, essa influência é considerada apenas nos termos de uma veneração de Boccaccio por Dante, sem que se tenha alguma vez questionado se Boccaccio não interviria para questionar, também, em função da própria poética, algumas das teses básicas do poema dantesco. Propõe-se precisamente essa tarefa, de tentar demonstrar como Boccaccio, ao intervir em tais teses, questiona seus princípios fundamentais, em primeiro lugar aquele do estatuto de verdade, a todo momento mencionado por Dante e proposto a seu leitor, e que Boccaccio submete a um exame atento, por vezes crítico. Isso se dá sobretudo em alguns trechos do Decameron, nos quais Boccaccio fala diretamente, sem a mediação dos próprios narradores (por exemplo, na Introdução à IV jornada); mas também pode ser identificado nas premissas e conclusões dos narradores às próprias narrativas. Nelas, a intangibilidade da verdade, e ao mesmo tempo a necessidade de interpretar a realidade, submetida a um constante escrutínio, propõe-se não como modelo oposto, mas decerto radical e problematicamente diferente daquele que a experiência dantesca sugere.

PALAVRAS-CHAVE: Decameron; Giovanni Boccaccio; Dante Alighieri; Francesco Petrarca; modelos narrativos; verdade; interpretação.

ABSTRACT: It is a well known fact that Boccaccio's The Decameron has been strongly influenced by Dante's The Divine Comedy and Petrarch's The Canzoniere. Yet, such an influence, particularly as Dante is concerned, is generally considered a symptom of Boccaccio's veneration for his great predecessor. Rarely have scholars asked themselves whether Boccaccio, following Dante's path, also disputes some fundamental points of Dante's poem. The article tries to demonstrate how Boccaccio faces this problem as well as how Boccaccio critically queries the fundaments of truth in the Divine Comedy. This can be conspicuously observed in parts of The 
Decameron where the author speaks directly to the reader (particularly in the Introduction to day IV). However, it can also be identified in other parts of the book, as it is the case with the assumptions and conclusions of the single tales. If not a totally opposite model to Dante's poem, these sections of Boccaccio's masterpiece have to be regarded as a radically different literary experience.

KEYWORDS: Decameron; Giovanni Boccaccio; Dante Alighieri; Francesco Petrarca; narrative models; truth; interpretation. 


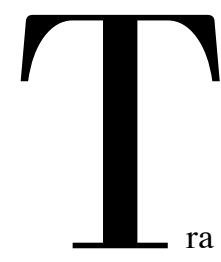

ra i progressi indubbi compiuti negli ultimi tempi dalla critica boccacciana va annoverata l'indicazione del carattere per così dire extra-novellistico della raccolta novellistica per eccellenza della letteratura italiana ed europea. Non è infrequente ormai vedere definire il Decameron come un "trattato" (è capitato anche a me), definizione che evidentemente non intende essere onnicomprensiva, tanto meno conclusiva, ma che ha forse il vantaggio di attrarre la nostra attenzione su aspetti fino a poco tempo fa trascurati (e non ancora divenuti base percettiva comune) del capolavoro di Boccaccio; tra essi, quello che lo qualifica appunto come un trattato (in forma narrativa) che ha per oggetto i mores, a cominciare dai mores concernenti l'amore (BATTAGLIA RICCI, 2013, p. 27-56). Senza pretendere di avanzare formulazioni definitive, si potrebbe affermare che il Decameron è un testo che, tramite complesse strategie 
narrative, affronta temi della filosofia morale del medioevo exeunte. A riconsiderare le cose anche sotto questo aspetto concorre innanzi tutto la mise en page dell'autografo che, come hanno sottolineato Teresa Nocita e Lucia Battaglia Ricci, presenta - tramite un'attenta scansione dei piani del discorso, contemplante l'adozione di lettere capitali di differente misura (di colore rosso e turchino) che scandiscono varie sezioni del testo (inizio delle giornate, incipit e parti liminari di ogni novella, conclusione delle singole giornate, ballate al termine di esse) -, una commatizzazione del dettato, tendente a conferire peso rilevante ai passi introduttivi di ogni racconto, a indicare che il racconto stesso tenderà a porsi in funzione dimostrativa (sia essa armonica o contrastante) nei confronti di quelli': tratto che, sia detto di sfuggita, è dato rilevare, sia pure senza che vi siano coincidenze perfette, anche negli altri codici della proto-diffusione (il cosiddetto frammento magliabechiano: Firenze, Biblioteca Nazionale Centrale, II II 8; ${ }^{2}$ Piacenza, Biblioteca Passerini Landi, cod. Vitali 26, testimone frammentario; Paris, Bibliothèque Nationale de France, Italiano 482) ${ }^{3}$. Tutto ciò a significare che, almeno nella diffusione del testo avvenuta entro la parabola biografica di Boccaccio, l'indicazione di lettura fornita da quella demarcazione iniziale voluta dall' autore è percepita come fatto distintivo del Decameron e delle sue strategie di presentazione - viceversa già nella prima diffusione è rilevabile una consistente obliterazione di quei tratti (CURSI, 2013, p. 109). Si tratta di spunto che anche il lettore del XXI secolo, che pure in nessun modo può rinunciare alle sue prospettive di lettura, deve tenere nel debito conto, se vuole, proprio nell'aprirsi di queste divaricazioni, conferire al testo la ricchezza che la storia della sua fruizione gli ha attribuito.

Apro una parentesi breve per rimarcare che quanto ammoniva a fare Barbi già nel 1927, cioè procedere a un confronto il più possibile completo tra tutti i testimoni del Decameron, si dimostra compito sempre più urgente (BARBI, 1973, p. 35-85). Avendo recentemente (in funzione di una monografia decameroniana cui sto attualmente lavorando) portato a termine una collazione completa tra il Parigino italiano e l'autografo Hamiltoniano, credo di poter affermare che il testo come oggi lo leggiamo, secondo la benemerita edizione di Branca (BOCCACCIO, 1980), è da rivedere in molti e non secondari punti, posto che, a prescindere dal noto fatto che gli autori sono sempre copisti inaffidabili di se stessi, Boccaccio, come proprio Branca ha più volte ricordato, esercita quella funzione in modo particolarmente trascurato e frettoloso, così che in numerosi passi quantomeno dubbi, e in altri luoghi certamente erronei, la lezione dell'Hamiltoniano va sanata, s'intende solo quando sia possibile escludere che il passo in discussione sia interessato da varianti d'autore, col ricorso al Parigino (ma naturalmente l'esame andrà allargato agli altri

1 Cfr. NOCITA, 1999; BATTAGLIA RICCI, 2000, 141-49; inoltre RAFTI, 2001, part. pp. 7-10; MALAGNINI, 2003. La commatizzazione è stata adottata nelle seguenti edizioni: HERNÁNDEZ ESTEBAN, 1994; FIORILLA, 2011; QUONDAM, FIORILLA, ALFANO, 2013.

2 Sulla peculiare natura di questo testimone cfr. Cursi (2007, p. 21-31), da integrare, in relazione all'identificazione del copista, con Cursi (2013, p. 111-112)

3 Per una puntuale illustrazione del sistema di paragrafatura nei codici della tradizione, in relazione alla proto e prima diffusione, cfr. Cursi (2007, p. 155-159). Per la fedeltà dei testimoni della proto-diffusione alla mise en page ideata da Boccaccio cfr. Cursi (2013, p. 110-128). 
testimoni importanti) ${ }^{4}$. Non si contano i casi di saut du même au même, errori di anticipo o di ripetizione, lezioni deteriori, che costellano l'autografo. Quanto poi al Parigino, ritengo che definirlo una prima redazione del Decameron, come sostenuto da Vitale e Branca (2002), sia senz'altro eccessivo: se si pone mente ai casi che spesso vengono citati per giustificare tale qualifica (Ariosto, Manzoni, ecc.), si potrà parlare al massimo di differente stesura (termine che va a mio avviso distinto per quanto possibile da quello di redazione), concetto che batte l'accento sul fatto che la fisionomia del testo non viene alterata in modo così cospicuo da far pensare a differenti stadi appunto redazionali: i quali sono caratterizzati, nell'iter testuale, da mutamento marcato (e spesso coerentemente esteso all'insieme) della facies linguistica (in senso fonetico e/o morfologico-sintattico), dislocazione di segmenti più o meno ampi dello scritto, espansione o condensazione del testo, eventuale differente seriazione dei pezzi (per opere in cui essi siano isolabili), ecc. Se si fa il confronto con quanto ci è dato conoscere dalle carte petrarchesche in relazione ai Rerum vulgarium fragmenta (che è forse il riscontro più congruo nella fattispecie), credo si possa dire che, non verificandosi tra il Parigino italiano e l'Hamiltoniano le condizioni appena richiamate, ci si trova, con i testimoni più importanti del Decameron, di fronte a una fenomenologia del tutto differente, cui non conviene la qualifica di diversa redazione. ${ }^{5}$

È consapevolezza diffusa che il Decameron sia il primo libro novellistico d'autore della nostra letteratura. Una prova precedente pur importante per lo stesso testo boccacciano come il Novellino denuncia, infatti, sin dal titolo apposto al manoscritto più antico (Libro di novelle e di bel parlare gientile), la sua natura di testo antologico, pur se in esso sia ipotizzabile rintracciare una qualche individualità autoriale: ${ }^{6}$ ma si tratta di autore che tira le fila di narrazioni preesistenti, non di autore che presiede in prima persona a un progetto letterario ambizioso, incomparabile coi tentativi antecedenti o coevi. Malgrado tale indiscutibile preminenza, è da tempo accertato che Boccaccio si sia valso di quel precedente, come di tanti altri disparati che poté conoscere (classici e medioevali), per imbastire un libro che, poggiato su tanti e diversificati materiali, non assomiglia ad alcun altro.

La sede per condurre un adeguato discorso sui modelli narrativi del Decameron è naturalmente un commento aggiornato a esso, ormai inderogabile: dico aggiornato perché quello storico e

4 Sulla questione mi limito a rimandare ai contributi più recenti e importanti (tutti con bibliografia pregressa): Breschi (2004); Fiorilla (2010, 2013). Si tenga presente che l'edizione di Quondam, Fiorilla e Alfano (BOCCACCIO, 2013) corregge già in più punti, facendo ricorso al Parigino e secondo le modalità indicate, il testo fissato da Branca.

5 Nell'impossibilità di fornire adeguato supporto bibliografico sul Petrarca volgare, mi limito a rimandare a Santagata (PETRARCA, 2004) e a Paolino (PETRARCA, 2000) (per la parte relativa al Canzoniere).

6 Cfr. la Nota al testo in CONTE 2001, pp. 267-93. 
tuttora indispensabile di Branca (BOCCACCIO, 1980) non ha sempre potuto tenere conto, nelle sue ultime versioni, delle molte novità che si sono accumulate sul capolavoro di Boccaccio, particolarmente in relazione al reperimento delle fonti, il cui spettro è amplissimo, e il cui utilizzo è eccezionalmente sofisticato; né d'altra parte la citata fresca edizione di Quondam, Fiorilla e Alfano (BOCCACCIO, 2013) non offre informazione alcuna su tale versante (se non per quanto in parte lodevolmente risarcito dalla Scheda introduttiva preposta da Alfano a ogni singola giornata). Tanto sofisticato quell'utilizzo, viene fatto di aggiungere, che a molti appare, a mio avviso a torto, che si insista troppo su questo snodo. Dando per scontato che il reperimento delle fonti sia un obbligo in sede di commento (ciò che non sempre avviene), non intendo con questo sostenere né la sicura identificabilità di quelle (che in Boccaccio, come in tutti gli autori culturalmente ricchi, quasi mai sono isolabili), né tanto meno conferire a quell'individuazione un valore risolutivo, come se la critica decameroniana trovasse in essa il suo massimo esercizio; anche perché l'autore interviene su più livelli, e in essi la testualità identificabile (quando ci sia) è solo una delle componenti. Ma conoscere come Boccaccio ha lavorato sui propri spunti, come li ha riorientati, presentando al lettore un testo eccezionalmente stratificato, in cui essi, interagendo coll'insieme, cooperano a rendere il Decameron un testo che ha mire ben diverse dal puro intrattenimento, non mi pare dettaglio trascurabile.

Se si sorvolasse su tali fatti, si agirebbe come non si sapesse quanto multiforme, e per tanti versi imprevedibilmente prensile, sia la cultura di Boccaccio. Certo, gli manca la solidità dottrinale e il rigore mentale di Dante, non è in possesso della sensibilità filologica e della inarrivabile cultura classica di Petrarca. Ma ciò nulla toglie alla sua forza di lettore non solo onnivoro ma acuto, capace di trarre dai testi le lezioni più impensabili: ciò che rende spesso difficile l'individuazione corretta delle sue fonti. Tornerò su questo punto. Per ora ritengo necessario soffermarmi su un fatto che dovrebbe apparire chiaro a molti, ma che deve ancora diventare coscienza comune: vale a dire la natura, estremamente composita, del libro, e di conseguenza dell'uso che esso fa dei suoi modelli. Fino a non molto tempo fa il cartiglio di testo d'intrattenimento apposto al Decameron ha messo in ombra il progetto alto del testo; anche per questo motivo uno sguardo attento all'autografo, messo in pagina alla stregua di un testo universitario, si mostra istruttivo. ${ }^{7} \mathrm{E}$, sia detto di sfuggita, la stessa prima fruizione dell'opera, che Branca ha collegato ad ambienti prevalentemente mercantili, è stata incanalata in più eterogenei e articolati percorsi dalle recenti analisi di Marco Cursi (2007), che ha sottoposto a vaglio autoptico 60 manoscritti del Decameron, dalla proto-diffusione (1360-1375) alla seconda diffusione (1426-1490): dalle sue ricerche risulta che i copisti per passione non possono essere

7 Su questo punto cfr. PETRUCCI 1983, pp. 514-17 
definiti figure prevalenti, ma che invece a essi si affianca una rilevante presenza di copisti $a$ prezzo, cioè di copisti professionali o semi-professionali, il che costringe a ripensare il problema della produzione e della circolazione dell'opera (CURSI, 2007, p. 134-142). Resta che, dando al suo capolavoro quella veste, Boccaccio ha inteso mettere sull'avviso i suoi lettori, che però hanno fatto fatica a intendere fino in fondo la radicalità delle sue mire, tanto più perché tale radicalità si pone sotto l'egida ironica dell'ingannevole semplicità di accesso. L'atteggiamento condiscendente storicamente spesso riservato al Decameron non deve stupire, se si pensa che, sin dalle fondazioni romanze, la narratio brevis stenta a farsi largo tra generi letterari dotati di maggior prestigio - tra tutti, l'epica e la lirica. La conoscenza dell'officina di Boccaccio consente oggi, moltiplicatisi gli studi in questa direzione, di apportare notevoli correzioni di rotta a questo erroneo avvicinamento al testo. ${ }^{8}$

Che tra i modelli un posto importante spetti anche a Petrarca pare indiscutibile, pure se una ricerca sistematica in questo senso deve ancora essere condotta. Certo, Branca ricorda giustamente nel suo commento come il sonetto introduttivo del Canzoniere deponga molti semi già nel proemio al Decameron, fino a riprese verbali (a cominciare dal primo giovenile errore petrarchesco e dalla prima giovinezza boccacciana). Sono però convinto che non è tanto o solo questo il territorio dove occorre cercare per trovare riverberi petrarcheschi attivamente operanti sul Decameron, che andranno cercati almeno in ugual misura nelle opere latine, nell'Africa come in alcune epistole del Familiares (VELLI, 1995, p. 237-243).

Ben diverso, ampiamente documentato e da diverse prospettive analizzato, il peso esercitato dal modello dantesco. ${ }^{9}$ Ma anche qui bisogna intendersi. Una volta allestito l'indispensabile regesto degli echi intertestuali si è solo all'inizio dell'opera, soprattutto quando si ha a che fare con un irraggiungibile genio della parodia come Boccaccio. Con lui occorre conferire al termine "parodia" il suo significato più ampio e incisivo di riscrittura sempre problematicamente atteggiata, dando per scontato che il Decameron offra anche esempi di parodia nel senso più comunemente accettato di abbassamento, appunto, parodico. Ma con Dante l'impegno di Boccaccio, per ragioni che non devono essere spiegate qui, è massimo, e si traduce, particolarmente nel Decameron, in un vero corpo a corpo colla Commedia. Va peraltro aggiunto che il suo atteggiamento nei confronti dell'intera opera di Dante registra, negli anni, alcune

8 Parte fondamentale di tale correzione di rotta spetta al riconoscimento, recentissimo, dei molti autografi boccacciani definibili come editoriali (per distinzione dalle edizioni d'autore autografe; cfr Battaglia Ricci, 2010, part. p. 123-128); cfr., per entrambe le tipologie, il regesto di Cursi e Fiorilla (2013).

9 Si pensi almeno agli studi di Hollander (1997) e alle indicazioni di Bettinzoli Cfr. HOLLANDER 1997; BETTINZOLI 1981-1982 e 1983-1984, cui va aggiunto almeno DELCORNO 1979. 
correzioni di rotta, da non enfatizzare né ignorare; ${ }^{10}$ poiché sta diventando di moda rintracciare, nell'attività letteraria di Boccaccio, una progressiva "dantizzazione", cui si accompagnerebbe un'altrettanto visibile "petrarchizzazione", suggerirei cautela e opportuno freno di fronte al rischio di seduzione esercitata da tali sirene. Per tanti secoli lettori tutt'altro che sprovveduti non avrebbero capito alcunché? Abbattere i falsi miti è sacrosanto, purché ci si astenga dal venerare frettolosamente i nuovi; tanto più se le nuove sirene non tengono sempre il dovuto conto della specificità del sistema letterario dell'ultimo Medioevo.

Per disporre le cose nella prospettiva corretta, va innanzi tutto rilevato come la posizione di Boccaccio in quanto autore, in rapporto al proprio testo, non possa essere comparata né con quella di Dante né con quella di Petrarca. Boccaccio non è né interno al suo libro in quanto auctor e insieme in quanto agens - come accade per Dante (CONTINI, 1970); né d'altra parte si può pensare che il suo distacco dalle tempeste amorose giovanili, secondo la mossa iniziale del proemio, possa essere presentato con lo stesso carattere esemplare di faticoso raggiungimento di una mutatio vitae sulla scorta agostiniana, così come capita per il Canzoniere (SANTAGATA, 2004, p. 41-99). La sua posizione rispetto al circolo dei narratori non gli concede potere testimoniale paragonabile a quello degli altri due grandi: i due brevi cenni, in I Intr., 16, 18, proprio nella loro cronachistica puntualizzazione, non permettono assimilazioni con l'enormità di quelle esperienze. Egli è piuttosto esterno al suo libro, e solo raramente si permette di intervenire ai suoi bordi, per difendere la propria opera, e per cercare di offrire le giuste prospettive di lettura. In altre parole, Boccaccio si cela dietro la voce dei narratori nel Decameron, mentre Dante, all'opposto, è sempre al centro della Commedia, e Petrarca ha saldamente in mano le redini del suo discorso penitenziale. Tuttavia proprio qui si nasconde un rischio di sottovalutazione dei procedimenti parodistici di Boccaccio. Hollander (1997, p. 21-52) ha definito il suo rapporto con Dante come "imitative distance" e, più in dettaglio, ha proposto di leggere la vicenda di ser Ciappelletto e la novella di frate Cipolla rispettivamente come riscrittura del canto di Brunetto (Inferno XV) e come parodia di Dante autore della Commedia. Forse le tesi di Hollander sono estremistiche (perché si possa parlare di riscrittura e parodia occorrono evidenze testuali che nei casi indicati mi paiono non sempre manifeste), e lo stesso studioso ammette che le sue prese di posizione possono essere considerate "over-ingenious" (Ibid., p. 27); ma la sostanza del suo discorso appare senz'altro condivisibile.

Credo si possa soprattutto sottoscrivere la sua affermazione secondo la quale allusioni e citazioni dalla Commedia sono poste nel Decameron sotto una lente non di rado critica. In effetti, non dobbiamo prestare troppa fede a Boccaccio allorché segue il percorso di Dante, poiché nella

10 Per un rapido orientamento su quest'ultimo punto mi limito a rimandare a Fumagalli (2013) (con bibliografia pregressa). Osservazioni pertinenti anche in Battaglia Ricci (2013, p 148-56). 
maggioranza dei casi egli non sta cercando una conciliazione o un accomodamento con il suo testo. Ritengo in questo senso che, una volta constatata l'entità del dialogo intertestuale che il Decameron intrattiene con la Commedia, ci si debba indirizzare a mettere in rilievo alcuni punti cruciali che sembrano, riscrivendoli, ridiscutere assunti centrali del testo dantesco.

Cominciamo dai dati più semplici e a tutti noti: il Decameron è costituito da cento racconti (in verità ce n'è un centunesimo, la novella delle papere, che ha un ruolo decisivo), così come i canti della Commedia. La data della grande peste, il 1348, cade nel trentacinquesimo anno d'età di Boccaccio, dunque nello stesso punto della parabola biografica di Dante autore della Commedia. L'insieme di tali dati rimanderebbe a una sorta di senso di ordine e simmetria comparabile a quello che si può ravvisare in Dante. E ciò è precisamente quanto molti autorevoli lettori decameroniani pensano. Il che inevitabilmente porta (e infatti ha portato) alla conclusione che, seguendo il ritmo ascensionale della Commedia, Boccaccio presenterebbe il suo libro come un cammino di faticosa progressione verso i valori morali più elevati. Ma ci si dimentica che tali valori sono sperimentati da Dante anche in quanto protagonista (agens), non solo in quanto autore (auctor); e che, per le ragioni su illustrate, la posizione di Boccaccio non è comparabile. Secondo la lettura a lungo dominante, Ciappelletto sarebbe il perfetto opposto di Griselda. Ciò che, con riguardo a quest'ultima novella, è vero per la storia, non per la voce (quella di Dioneo) che la racconta; è vero per l'enunciato, non per l'enunciazione. Lo dimostra la beffarda chiosa del narratore alla conclusione della vicenda:

Che si potrà dir qui? se non che anche nelle povere case piovono dal cielo de' divini spiriti, come nelle reali di quegli che sarien piú degni di guardar porci che d'avere sopra uomini signoria. Chi avrebbe, altri che Griselda, potuto col viso non solamente asciutto ma lieto sofferir le rigide e mai piú non udite prove da Gualtier fatte? Al quale non sarebbe forse stato male investito d'essersi abbattuto a una che quando, fuor di casa, l'avesse in camiscia cacciata, s'avesse sí a un altro fatto scuotere il pilliccione che riuscito ne fosse una bella roba (X 10 68-69).

Tutto ciò non si legge ovviamente nella versione della storia fornita da Petrarca, che, infatti, benché all'ignaro delle nostre facili distinzioni teoriche, scrive, nell'epistola a Boccaccio che accompagna la traduzione latina (Seniles, XVII 3): "Historiam tuam, meis verbis explicui" (PETRARCA, 2006, p. 540), con ciò mostrando di capire acutamente la divaricazione tra 
enunciato ed enunciazione. Tornando al rapporto con Dante, dobbiamo tenere a mente l'ammonimento di Hollander, per il quale Boccaccio sottopone a scrutinio due postulati fondamentali della Commedia: la veridicità poetica e la sua applicabilità morale. Non solo Dante, come si vedrà, è implicato in questa rete di rimandi intertestuali o interdiscorsivi, ma non c'è dubbio che sia lui a essere uno dei bersagli principali della riscrittura boccacciana. Tale riscrittura si situa a diversi livelli, dei quali ritengo importante analizzare alcuni tra i risvolti più elusivi.

L'elusività deve d'altra parte fare i conti anche con una difficile allusività, che di quella costituisce ingrediente essenziale. Mi spiego, limitandomi a indicare un solo caso, che risulta però di portata ampia ed estensibile in realtà su tutto il testo. Per secoli il Decameron è stato letto come un libro incitante al piacere erotico e sessuale, il che ha dato la stura all'abuso del termine "boccaccesco" (che qui, per buone ragioni, non si adotterà), usato in funzione esclusiva da chi, evidentemente, non ha mai riflettuto, come occorre fare, al libro che ha per titolo Decameron, ma ha scorso semplicemente le storie più esposte su quel versante. Versante che naturalmente esiste, ma che è una componente del libro, componente che, soprattutto, deve essere tenuta, come altre, nel conto delle strategie di intervento della letteratura sulla realtà che il proprio Boccaccio ha inaugurato; ma che, precisamente per il fatto di incardinarsi in tali strategie, non può aspirare ad avere rappresentanza dominante, meno che mai esclusiva. So bene che sottovalutare il coté erotico-sessuale sarebbe errore madornale, perché andrebbe in un certo senso contro la logica del libro. Ma contro essa andrebbe anche una lettura che stentasse a rendersi conto del fatto che, precisamente in relazione al suo relativismo procedurale, il testo si affida, per la sua piena intelligenza, all'utilizzo che ne può fare il lettore, lasciato unico responsabile della faccenda; dunque, se di cammino verso i valori moralmente elevati si deve trattare, è il lettore (emulo in questo senso dei narratori della compagine) a doverne essere sperimentatore, conoscendo il libro intus et in cute. È da vedere qui, nella centralità della figura del lettore, uno dei massimi punti di distanza da Dante, che pure il lettore continuamente invoca (BATTAGLIA RICCI, 1987, p. 191-196). Ma la direzione è in un certo senso inversa, perché il lettore è in Dante esortato a credere al racconto del viaggio anche nei suoi risvolti meno credibili ("Sempre a quel ver c'ha faccia di menzogna / de' l'uom chiuder le labbra fin ch'el puote, / però che sanza colpa fa vergogna; / ma qui tacer nol posso; e per le note / di questa comedía, lettor, ti giuro", ecc.; Inf., XVI 124-28), e a effettuare di conseguenza il necessario percorso di purificazione; mentre in Boccaccio il lettore è di fatto lasciato libero nell'effettuare il suo percorso di lettura, persino, giusta quanto afferma la Conclusione dell'autore (15), evitando racconti che possano 
non interessargli o sembrargli sconvenienti (col risultato negativo, s'intende, di collocarsi tra i lettori non all'altezza del testo).

Il Decameron ha quello che oggi chiameremmo (non molto appropriatamente) un sottotitolo, giacché è "cognominato principe Galeotto" (e tale denominazione si ripete alla conclusione del libro). Non esiste qualifica più potenzialmente fuorviante di questa, ciò che è infatti comunemente accaduto nelle pratiche di lettura. Perché, avendo la memoria alle parole di Francesca, "Galeotto fu 'l libro e chi lo scrisse" (Inf., V 137), e alla loro banalizzazione, per la verità fatta propria dallo stesso Boccaccio commentatore di Dante, potrebbe accendersi un cortocircuito che il testo viceversa non autorizza in alcun modo, e anzi dimostra essere completamente erroneo. Queste le parole di Boccaccio nelle sue Esposizioni sopra la Comedia di Dante, a proposito del passo ricordato:

E così vuol questa donna dire che quello libro, il quale leggevano Polo ed ella, quello officio adoperasse tra lor due che adoperò Galeotto tra Lancialotto e la reina Ginevra; e quel medesimo dice essere stato colui che lo scrisse; per ciò che, se scritto non l'avesse, non ne potrebbe esser seguito quello che ne seguì. Quel giorno più non vi leggemmo avante. Assai aconciamente mostra di volere che, senza dirlo essa, i lettor comprendano quello che dell'essere stata basciata da Polo seguitasse. (PADOAN, 1965, V (1) 184, p. 324).

Paradossalmente, si può dire che la stessa banalizzazione operata da Boccaccio rafforzi il senso della distanza su cui richiamo l'attenzione. All'inizio del Decameron (I Intr., 79), Boccaccio ricorda che Panfilo, Filostrato e Dioneo, i tre narratori maschili, hanno, tra le sette narratrici (Pampinea, Fiammetta, Filomena, Emilia, Lauretta, Neifile, Elissa), le loro donne; di più, un rapido inciso, che rompe la scorza convenzionale delle iniziali schermaglie dialettiche, registra che Neifile, all'atto del primo incontro con il terzetto dei giovani, "tutta nel viso divenne per vergogna vermiglia per ciò che l'una era di quelle che dall'un de' giovani era amata" (I Intr., 81). Ebbene, sono molti i luoghi del testo che ribadiranno il filtro interposto da Boccaccio tra letteratura e vita. Basta qui solo, a chiarire ciò che sopra è stato detto sulle strategie d'autore, quanto espresso da Panfilo (il narratore maschile cui sembra affidata, per così dire, la posizione di autorità che tra le donne spetta a Pampinea), a conclusione della giornata da lui retta, non per caso la decima, così come la prima è governata appunto da Pampinea: 
Noi, come voi sapete, domane saranno quindici dí, per dovere alcun diporto pigliare a sostentamento della nostra santà e della vita, cessando le malinconie e ' dolori e l'angoscie, le quali per la nostra città continuamente, poi che questo pistolenzioso tempo incominciò, si veggono, uscimmo di Firenze; il che, secondo il mio giudicio, noi onestamente abbiam fatto; per ciò che, se io ho saputo ben riguardare, quantunque liete novelle e forse attrattive a concupiscenzia dette ci sieno, e del continuo mangiato e bevuto bene, e sonato e cantato (cose tutte da incitare le deboli menti a cose meno oneste), niuno atto, niuna parola, niuna cosa né dalla vostra parte né dalla nostra ci ho conosciuta da biasimare: continua onestà, continua concordia, continua fraternal dimestichezza mi ci è paruta vedere e sentire; il che senza dubbio in onore e servigio di voi e di me m'è carissimo (X Concl., 3-5).

L'insistenza sul mantenimento dell'onestà (termine chiave del libro, coi suoi derivati), a dispetto di occasioni che avrebbero potuto facilitare deroghe a essa (ciò che fanno solo "deboli menti", evidentemente inadatte a intendere la lezione del Decameron), non potrebbe risultare più chiara. ${ }^{11}$ Boccaccio, per bocca di Panfilo, ci comunica che, per tutta la durata del loro intrattenimento, narratori e narratrici della raccolta, e tanto più quelli già implicati in legami amorosi, non hanno commesso l'errore degli amanti danteschi (che risultano così essere una sorta di declinazione negativa, o mimeticamente ingenua, delle forze agenti nel libro “chiamato Decameron"): non sono stati insomma vittime, come Francesca e Paolo, dell'indebita sovrapposizione tra letteratura e vita (BATTAGLIA RICCI, 1987, p. 179-198). Davvero, mai etichetta fu escogitata con maggiore sottigliezza e insidia di quella usata da Boccaccio nel dare la qualifica di "Galeotto" al suo libro; la responsabilità del cui uso corretto deve ricadere, per esplicita presa di posizione di Boccaccio (consegnata appunto alla Conclusione dell'autore), senz'altro sul lettore. Mi spiego: facendo ricorso alla classica distinzione di Berlin (1998, p. 69157) tra il riccio e la volpe, si può dire che se il primo fa convergere tutto in una visione centrale, forzando così il lettore al consenso, la seconda lo lascia libero, disseminando il proprio percorso di false tracce, mettendolo anzi spesso fuori strada, costringendolo faticosamente, novello Pollicino, a ritrovare da solo la retta via (e questa è senz'altro la posizione di Boccaccio).$^{12}$

Su un diverso piano, che chiama in causa apertamente l'allusività intertestuale, si pone l'introduzione alla quarta giornata: uno dei segmenti testuali che, ricadendo sotto l'esclusiva

$11 \mathrm{Su}$ tale punto cfr. l'esaustiva rassegna (dal mondo antico a Boccaccio, e oltre) di Cherchi (2004).

12 La distinzione, che ho sommariamente ripreso dalla ben diversamente articolata formulazione originaria, in BERLIN 1998²: 69-157. 
pronuncia d'autore, e sottraendosi dunque alle intermediazioni dei narratori, implicano un coefficiente alto di esplicitezza. Ebbene, anche in questa fondamentale porzione del libro la sfida con Dante è nettamente percepibile e accentuata (e resa problematica) da specifici agganci testuali. Leggiamone due brevi tratti, desunti dall'inizio e da un brano che si dispone verso la fine della "autodifesa":

Carissime donne, sí per le parole de' savi uomini udite e sí per le cose da me molte volte e vedute e lette, estimava io che lo 'mpetuoso vento e ardente della invidia non dovesse percuotere se non l'alte torri o le piú levate cime degli alberi; ma io mi truovo dalla mia estimazione ingannato. Per ciò che, fuggendo io e sempre essendomi di fuggire ingegnato il fiero impeto di questo rabbioso spirito, non solamente pe' piani ma ancora per le profondissime valli mi sono ingegnato d'andare; il che assai manifesto può apparire a chi le presenti novellette riguarda, le quali non solamente in fiorentin volgare e in prosa scritte per me sono e senza titolo, ma ancora in istilo umilissimo e rimesso quanto il piú si possono. Né per tutto ciò l'essere da cotal vento fieramente scrollato, anzi presso che diradicato e tutto da' morsi della 'nvidia esser lacerato, non ho potuto cessare; per che assai manifestamente posso comprendere quello esser vero che sogliono i savi dire, che sola la miseria è senza invidia nelle cose presenti (IV Intr., 2-4).

E volendo per questa volta assai aver risposto, dico che dallo aiuto di Dio e dal vostro, gentilissime donne, nel quale io spero, armato, e di buona pazienza, con esso procederò avanti, dando le spalle a questo vento e lasciandol soffiare; per ciò che io non veggio che di me altro possa avvenire, che quello che della minuta polvere avviene, la quale, spirante turbo, o egli di terra non la muove, o se la muove, la porta in alto, e spesse volte sopra le teste degli uomini, sopra le corone dei re e degli imperadori, e talvolta sopra gli alti palagi e sopra le eccelse torri la lascia; delle quali se ella cade, più giù andar non può che il luogo onde levata fu (ivi, 40). 
Le tessere dantesche (soprattutto l'esortazione di Cacciaguida, Par., XVII 133-35) di questi e di altri passi dell'introduzione alla quarta giornata sono tutte illustrate da Branca nel suo commento, e perciò non è necessario insistervi, se non per rilevare che quelle tessere naturalmente hanno la funzione di esaltare, contro ogni atteggiamento ufficioso di modestia, la propria opera. Mi concentrerò invece su un'immagine ricorrente e centrale dei lacerti selezionati, quella del vento, sollevato dall'invidia; non va però dimenticato che l'invidia, divenuta attiva, viene veicolata anche dalle figure del morso e dei denti, secondo una traiettoria che ha precedenti tanto veterotestamentari che classici. ${ }^{13}$ Quanto alla figuralità legata al vento, essa è radicata nella tradizione biblica (rimarco la cosa perché, diversamente da quanto accade per Dante e Petrarca, questo cartello è passato per lungo tempo inavvertito negli studi boccacciani) ${ }^{14}$; il suo uso è soprattutto collegato a tre differenti situazioni, due delle quali implicanti la potenza di Dio, che si può esplicare: 1) o negativamente, a manifestazione della sua ira (Ex., X 13; Is., XVII 13; Jer., XVIII 17); 2) o positivamente, nell'aiuto concesso agli Israeliti nell'attraversamento del mar Rosso (Ex., XIV 21). Interessante in questo senso notare che, nel Nuovo Testamento, la presenza di Cristo è spesso associata alla calma delle acque in assenza di vento, risultando anche tale particolare come esito del nuovo patto stretto da Dio con l'uomo (Mt., VIII 26, XIV 32; Mc., IV 39, VI 51; Lc., VIII 24-25, ecc.); 3) l'immagine vale invece solo negativamente, a designare la vanità, tanto in fatti che in parole, della vita umana (Job, VI 26, VII 7; Ecclesiastes, $\mathrm{V} 15$, ecc.). Tale uso è fatto proprio da Dante nello svilimento della fama mondana che si legge nelle parole di Oderisi da Gubbio (Purg., XI 100-2). Va osservato che Boccaccio, nei lacerti su riportati, segue questo ultimo canale figurale, con ciò tacciando di vanità non tanto solo la propria fama (che pure, seguendo l'esempio dantesco, è anch'essa segnata da vanità, in una visione millenaristica), quanto anche il tentativo di sminuire il valore del Decameron.

A commento del secondo dei due passi selezionati (a proposito dell'azione del vento che, agendo sulla polvere "sopra le eccelse torri la lascia") Branca (BOCCACCIO, 1980, p. 469) chiosa: "le calunnie [...] non possono abbassare la sua opera, ma soltanto probabilmente innalzarla". La chiosa è giusta, naturalmente, ma forse si può andare più vicino al segno. Nel

13 Per le occorrenze veterotestamentarie cfr. Job, XVI 10; Ps., XXXIV 16, XXXVI 12, e soprattutto LVI 5, CXXIII 6, ecc. I precedenti classici rimandano soprattutto a Ovidio e Seneca, già adibiti alla funzione nella Fiammetta, V 19 , IX 19; cfr. Delcorno 1994, p. 119-120, 188, p. 326 n. 40, p. 389 n. 48.

14 Cfr. Bragantini (2012, p. 69-87), che qui in parte riprendo e sviluppo. Anche questo fondamentale fronte si sta positivamente aprendo: cfr. Battaglia Ricci (2013, p. 134-156), con indicazione anche di altri lavori in corso sul punto. 
primo passo selezionato, Boccaccio dice di essersi sforzato di percorrere le "profondissime valli", dato che il vento sollevato dall'invidia colpisce soprattutto "l'alte torri o le più levate cime degli alberi'. Ma, tornando sul punto alla conclusione dell'autodifesa, l'immagine, rimodulando forse Eccl., XII 7 ("et revertatur pulvis in terram suam unde erat" - "e ritorni la polvere alla terra, com'era prima"), in qualche modo si converte nel suo contrario. Proprio la inconsistenza che caratterizzerebbe il suo libro può garantirgli di arrivare sotto forma di polvere (altra immagine evidente di vanità) persino sopra le teste dei re e degli imperatori. Dunque l'iniziale protesta di aver evitato di situarsi in zone troppo rarefatte si converte nel suo contrario. Quanto a dire, se si tiene a mente da quale passo dantesco muova l'autodifesa, che Boccaccio rovescia il senso della voce profetica di Dante; per meglio dire, alterando i rapporti connessi al significante, lascia intatto il significato. Egli non è Dante, il vento di denuncia "che le più alte cime più percuote" (Par., XVII 134), ma è piuttosto mosso da esso; tuttavia la leggerezza del suo libro, come della sua persona, su cui insisterà la Conclusione dell'autore (23), garantisce anche a lui sufficiente libertà per esercitare la sua critica tanto verso i potenti della terra quanto verso i suoi oppositori.

Ho accennato alla scarsa attenzione fino a poco tempo fa riservata agli echi biblici nel Decameron. Dico echi perché parlare di intertestualità per il Vecchio e il Nuovo Testamento in relazione ad autore medioevale è usare termine che non designa con chiarezza il tipo di rapporti che vorrei illustrare. Mi servo qui delle parole di padre Pozzi (1996, p. 150), usate per Petrarca, ma perfettamente adoperabili per Boccaccio (e a maggior ragione, s'intende, per Dante):

Allora come oggi, ma ben più allora di oggi, le presenze bibliche non si risolvono, una volta allestito il catalogo delle citazioni, nella prospettiva del trapasso da testo a testo. La Bibbia era da una parte il libro (e non un libro pur massimamente autorevole), e dall'altra agiva fuori della misura libresca, in quanto era esperienza.

Fatta questa premessa, occorre specificare che tali echi non hanno, come è facile capire, nel Decameron lo stesso peso né (è questo ciò che più conta) la stessa evidenza che essi attingono nel Canzoniere o nella Commedia. Se è agevole risalire, per esplicita dichiarazione d'autore, da una sentenza ("quando pur sopravenisse il bisogno, io so, secondo l'Apostolo, abbondare e necessità sofferire"; IV Intr., 38) alla sua origine neotestamentaria ("Scio et humiliari, scio et abundare, ubique et in omnibus institutus sum et satiari et esurire, et abundare et penuriam pati"; Ad Philipp., IV 12), meno lo è reperire, dietro la voce inaugurale di Panfilo, nella novella di ser Ciappelletto ("Convenevole cosa è, carissime donne, che ciascheduna cosa la quale l'uomo fa, 
dallo ammirabile e santo nome di Colui, il quale di tutte fu facitore, le dea principio"; I 1 2), la stessa pronuncia paolina ("Omne quodcumque facitis in verbo aut in opere, omnia in nomine Domini Iesu, gratias agentes Deo et Patri per ipsum”; Ad Col., III 17) ${ }^{15}$. E infatti il rilievo stenta a trovare luogo nei commenti, non apparendo, salvo errore, nei moderni. Eppure sembrano esserci pochi dubbi che, in forza della sua posizione incipitaria nell'esercizio del racconto, quelle parole si ricolleghino alla sentenza paolina, così operante non solo come richiesta di sostegno divino, ma come augurio per il successo dell'impresa.

Insisto sulla difficoltà di reperire nel Decameron tessere intertestuali che rimandino, più o meno esplicitamente, alla Scrittura. Ma insisto anche sulla necessità di ogni sforzo per individuarle, allargando il discorso nella direzione più ardua dei commenti biblici, nonché degli scritti dei padri e dei dottori della Chiesa, tramite l'allegazione di un unico specimen. Trascrivo questo passo di san Bernardo p. 315c-d) - autore carissimo a Boccaccio -, passo a sua volta tessuto su Lamentationes, III 27-28:

"ut in coelum ascendas, necesse est levare te super te, calcando carnalia desideria quae in te militant adversus te" (In ascensione Domini, Sermo IV, De duabus malis ascensionibus, daemonis et primi hominis: et sex bonis, Christi et nostris). ${ }^{16}$

Suggerisco, con beneficio di dubbio, la possibilità che il brano sia ripercorso, con abrasiva parodia e mutazione di registro (passaggio dal serio al comico), nella decisione di Alatiel, donna dal silenzio sospetto, di cedere alle lusinghe di Pericone: "avvisandosi ['la donna'] che a lungo andare o per forza o per amore le converrebbe venire a dovere i piaceri di Perdicon fare, con altezza d'animo propose di calcare la miseria della sua fortuna" (II 7 23). Il travaso non si può definire scorrevole, se si eccettua l'uso di calcare (che è occorrenza unica nel Decameron), che viene a capovolgere l'esortazione di Bernardo, giacché lo stesso verbo lì usato a vincere il desiderio carnale viene adibito a una situazione perfettamente antitetica. Per il resto Boccaccio adotta le seguenti procedure: a) passaggio dalla seconda alla terza persona, con aggancio al modulo biblico, e recupero e dislocazione ("quivi tutta sola si vedeva"; "quivi sola senza aiuto o consiglio d'alcun si vedea": II 7 16,43) della condizione ivi esplicitata di solitudine e silenzio ("Sedebit solitarius et tacebit, quia levavit super se" - Lamentationes,

$15 \mathrm{E}$ tutto quello che fate in parole ed opere, tutto si compia nel nome del Signore Gesù, rendendo per mezzo di lui grazie a Dio Padre.

16 Il passo in Patrologia Latina, vol. 183, p. 315c-d. Per poter salire al cielo, è necessario che ti levi sopra di te, calpestando i desideri carnali che in te stesso combattono contro te (Sull'Ascensione del Signore, Sermone IV: Su due malevole ascensioni, del demonio e del primo uomo; e su sei buone, di Cristo e nostre). 
III $28^{17}$ ); b) ricorso, rispetto a Bernardo, a formula nominale (levare te super te>con altezza d'animo) per significare innalzamento e vittoria su sé di segno rovesciato; c) eliminazione della repetitio pronominale. Il procedimento qui attuato è propriamente il centone, perché preleva unità riconoscibili adeguandole a un contesto radicalmente altro rispetto a quello di partenza. Neppure sarà da considerare estranea la memoria, anche in tal caso capovolta, di Ovidio, Amores, III 11 5: "vicimus et domitum pedibus calcamus amorem" ${ }^{18}$. Va ancora rimarcato che il passo di san Bernardo su ricordato deve essere ben presente anche a Petrarca se, stavolta adottandolo in pieno (restando cioè nell'ambito del medesimo registro, dal serio al serio, solo spostandone l'area di applicazione), lo utilizza due volte, per di più in collegamento con altri sintagmi riconducibili alla stessa area frequentata da Boccaccio (nei passi subito oltre allegati, alto animo e "con altezza d'animo" sono sovrapponibili). Nella consolatoria Familiaris VI 3 a Giovanni Colonna al destinatario viene rivolto l'invito seguente:

meminisse laborum ac dolorum quos pro nobis Cristus pertulit [...]; meminisse clavorum ac lancee preciosissimique sanguinis, quo lavacro, sordibus ablutis, recreati et clementer admoniti sumus temporales alto animo calcare molestias, nichilque nisi eterne damnationis et penarum immortalium supplicia formidare. (PETRARCA, 2004-2009, II p. 828) ${ }^{19}$.

Ancora, in altra consolatoria, la Familiaris XVI 6 (stavolta al vescovo Nicolò da Viterbo), si legge: "Hinc iam virilter admonendum es, ut memineris per te ipsum mortalia ferre alto animo atque despicere, calcare presentia, sperare meliora [...]" (PETRARCA, 2004-2009, IV p. 2250). ${ }^{20}$

I passi sin qui allegati andranno naturalmente inseriti in un'argomentazione maggiormente serrata, e il più possibile documentata, per controllare se il centone di Boccaccio, nel passo in questione, non assimili, come credo possibile, anche il Petrarca delle Familiares appena citato (almeno quello del primo esempio, il secondo essendo troppo prossimo alle date più

17 Sieda costui solitario e resti in silenzio, poiché egli glielo ha imposto.

18 Ho vinto e calpesto sotto i piedi Amore domato.

19 Ricordarsi degli affanni e dei dolori che Cristo sopportò per noi [...]; ricordarsi dei chiodi, e della lancia e del preziosissimo sangue, il cui lavacro cancellò le nostre macchie, e ci rinnovò, e benevolmente ci ammonì a conculcare con animo nobile le temporali molestie, e null'altro temere, se non i tormenti di un'eterna dannazione e di pene senza fine. Datata Valchiusa, 30 maggio, l'epistola viene assegnata al 1342.

$20 \mathrm{Ma}$ ora debbo virilmente ammonirti a sopportare con animo nobile e a sprezzare le cose terrene, a non curare il presente, a sperare il meglio [...]. L'epistola è non datata, ma il consistente primo getto autografo trasmesso dal codice degli abbozzi reca la data del 15 febbraio 1353, e la copia nella raccolta è immediatamente a ridosso (cfr. PETRARCA, 2000, p. 112-114). 
basse ipotizzate per la composizione del Decameron $)^{21}$; ma intanto si potrà asserire che la formulazione usata da Boccaccio ("con altezza d'animo propose di calcare la miseria della sua fortuna”), che a noi può oggi apparire legata per troppo sottili fili alla rielaborazione operata da san Bernardo su Geremia, avrà avuto ben più alta possibilità di essere colta nella sua allusività dai primi (e, si capisce, più smaliziati) lettori del Decameron, e magari di Petrarca. Da tutto ciò si ricaverebbe, infine, una piccola, ma non trascurabile chiarificazione ermeneutica: qualora il passo boccacciano risultasse effettivamente esercizio centonario su Geremia, Lamentazioni e san Bernardo, via Petrarca, si avrebbe la definitiva conferma che in esso calcare vale "calpestare" (come a mio parere è indubbio), piuttosto che, come anche ritenuto possibile, "percorrere tutta intera l'infelicità delle sue [di Alatiel] sventurate condizioni”.

Altro scaffale relativo ai modelli che solo recentemente è stato messo nella giusta luce, principalmente per merito del compianto Giuseppe Velli, è quello dei classici. Si tratta di fatto sul quale è necessario soffermarsi perché la qualifica di libro d'intrattenimento conferita troppo sbrigativamente al Decameron ha messo la questione in prospettiva distorta. L'enfasi posta sulla contemporaneità degli eventi e dei protagonisti (come se questi ultimi non fossero tali anche nella Commedia, che pure coi classici ha commercio continuo) ha indotto a trascurare il fatto che Boccaccio, anche quando legge accadimenti e personaggi coevi, lo fa insistentemente col soccorso dei classici latini. Eppure, tale fatto non sembra ancora essersi tramutato in consapevolezza comune (e infatti ne mancano quasi sempre tracce nei commenti). Ma come interpretare la sdegnosa battuta di Guido Cavalcanti alla brigata che tenta invano di stringerlo d'assedio ("Signori, voi mi potete dire a casa vostra ciò che vi piace"; VI 9 12), se non col rimando alla sentenza senecana "otium sine litteris mors est et hominis vivi sepultura"22 (Epistulae ad Lucilium, X 82 3). ${ }^{23}$ La mancata registrazione della cosa negli attuali commenti stupisce ancor più se si riflette che le epistole di Seneca, ovviamente notissime a Boccaccio, sono anche oggetto di un'attenta opera di selezione antologica nello Zibaldone Magliabechiano autografo. Né costituisce impedimento il fatto che tale selezione si arresti ai primi sei libri, poiché si deve ritenere che all'atto dell'allestimento dello Zibaldone egli non possedesse ancora il codice di Seneca segnalato nel catalogo della "parva libraria" (COSTANTINI, 1974).

Con la risposta di Guido siamo ancora in territorio relativamente agevole. Più ardue le cose si fanno quando Boccaccio, che è mirabile contaminatore di generi e percorsi narrativi divergenti,

21 Su alcune traiettorie Petrarca-Boccaccio cfr. Velli (1995, p. 237-243), che indica tessere petrarchesche anche per la descrizione iniziale della peste (Epystole metrice, I 14, Ad se ipsum), e per alcuni passi della novella di Ghismonda (Africa, V 720-34).

22 L'ozio non nutrito di studi equivale alla morte, all'essere sepolto vivo.

23 Cfr. Velli (1991, 1995). Diversa trafila è individuata in Quondam, Fiorilla e Alfano (BOCCACCIO, 2013, part. p.970-971); ma la spiegazione del motto offerta da Betto Brunelleschi indica come determinante soprattutto il filtro senecano. 
decide di rifarsi a modelli che non si suppone possano trovarsi dietro il suo esercizio. Prendiamo ad esempio (si tratta ancora di una trouvaille di Velli) la novella di Bernabò e Zinevra. Il geniale innesto mercantile ha spiazzato i lettori, impedendo loro di intendere che lo spunto iniziale è offerto dalla narrazione del dopocena dei figli del re Tarquinio. Infatti “avendo [i 'mercatanti'] una sera fra l'altre tutti lietamente cenato, cominciarono di diverse cose a ragionare, e d'un ragionamento in altro travalicando pervennero a dire delle lor donne, le quali alle lor case avevan lasciate" (II 9 4), risponde precisamente a "Forte potantibus his apud Sex. Tarquinium [...] incidit de uxoribus mentio"24 (Livio, Ab urbe condita, I 57 6) (VELLI, 1995, p. 233-234). Dopodiché la narrazione va per la sua strada, non senza altri apporti, ancora, biblici, giacché le lodi tributate da Bernabò alla moglie sono tramate, come ho altrove illustrato, sull'elogio della mulier fortis che chiude i Proverbia (XXXI 10-31) (BRAGANTINI, 2012, p. 72). Ma aver ignorato, fino agli scavi di Velli, il precedente liviano (e continuare, in molti casi, a ignorarlo), ha senz'altro impoverito l'esercizio narrativo, e tolto molto alla sostanza antropologica (insieme biblica e classica, in perfetto parallelismo stavolta con Dante e Petrarca) di Zinevra; aggiungo che non ha messo nel giusto rilievo la pochezza della figura di Bernabò che, dall'implicito confronto con Collatino, viene ulteriormente immeschinita, per aver scommesso denari su una figura da lui stesso definita impareggiabile. Senza dubbio la difficoltà nel percepire il precedente liviano deriva, oltre che dalla decisione boccacciana di riorientare in ambito mercantile un episodio tra i più celebri dell' antica storia di Roma, dall'esito felice della narrazione. Ma appunto qui si cela un insegnamento decisivo in relazione alle modalità della riscrittura boccacciana. Essa infatti non si limita a ripercorrere precedenti testuali (di cui, per di più, sfrutta pochi tratti, e cancella quasi sistematicamente le tracce), ma ne sottopone a nuova lettura gli assunti, intellettuali o morali.

Torna qui utile avanzare un principio generale, che lo studio del trattamento delle fonti in Boccaccio suggerisce, non solo per ragioni attinenti alla fisionomia del libro: contrariamente a quanto succede col Dante della Commedia, nel quale insieme e particolari si compenetrano e rispondono con piena e reciproca evidenza, con Boccaccio (la stessa cosa può essere in buona parte sostenuta per il Petrarca del Canzoniere) la legge che governa l'insieme si può attingere solo dall'intelligenza del funzionamento del particolare.

Per fornire evidenza del fatto mi soffermo su alcune novelle della seconda giornata. Si cominci col proverbio che chiude la notissima novella di Alatiel: "Bocca basciata non perde ventura, anzi rinnuova come fa la luna" (II 7 122). ${ }^{25}$ Il detto proverbiale contraddice alla lettera la premessa di Panfilo ("mi piace di raccontarvi quanto sventuratamente fosse bella una saracina"; 7); ma l'intero segmento introduttivo è in realtà un'esposizione en raccourci della decima

24 Una volta, mentre stavano bevendo nella tenda di Sesto Tarquinio [...] il discorso cadde sulle mogli. 25 Per la funzione del proverbio nella narrativa dei primi secoli cfr. Bragantini (2014). 
satira di Giovenale, infarcita di sentenze morali tra le più note della latinità, e sottoposta a una lettura versipelle in chiave di trasgressiva sessualità, parallela e convergente col sistematico ribaltamento dello schema del romanzo alessandrino ${ }^{26}$. In questo caso pare arduo individuare se sia nato prima l'uovo o la gallina, se cioè il proverbio conclusivo abbia agito da spinta iniziale, o se esso sia la beffarda glossa terminale dell'indubbio spunto giovenaliano. Nel primo caso si dovrebbe pensare a un movimento di espansione attorno al nucleo proverbiale, nel secondo a una rielaborazione di diverso tipo, che ha di mira la riscrittura delle sentenze reperibili in Giovenale e termina con la loro smentita. Ma forse sarebbe più corretto pensare a un alternato movimento di sistole (riscrittura, tramite selezione, compressione, dislocazione, di Giovenale) e diastole (espansione dello spunto proverbiale, che smentisce la fonte). Allargando il panorama sarà forse possibile gettare uno sguardo più articolato non solo sull'officina letteraria di Boccaccio, ma sul suo modo di procedere in casi consimili. Si sa come la sua sopraffina pratica di parodista gli suggerisca la dislocazione di sequenze che hanno una fonte comune, e insomma come egli distribuisca su porzioni diverse del libro un medesimo spunto, variandone le componenti - il procedimento è stato illustrato con particolare acume da Forni (1992, 2008, p. 27-40). Una lezione ricavabile dalla pratica in prima persona del commento si potrebbe, grosso modo, formulare nei termini seguenti: tanto meno accorto e colto è l'autore da commentare, tanto più, una volta individuato un centro di irradiazione delle fonti, la strada si fa facile, perché da quel centro si dipartono generalmente anche i percorsi laterali. Nel caso opposto, di autore acculturato e abile nel dissimulare le tracce, la situazione è antitetica: aver trovato una sicura fonte non può offrire alcuna garanzia, non solo perché tale fonte non è esclusiva, ma perché non è detto che non balzi di nuovo fuori dove meno ce la si può aspettare.

Si torni sulla decima satira di Giovenale: essa si concentra sulle assurde richieste che gli uomini fanno agli dei, pregandoli di ottenere ricchezza, potere, bellezza, tutti e sempre fonti di sciagure. Sarebbe meglio chiedere, scrive Giovenale, nient'altro che mente e corpo sani. L'esortazione che chiude la satira è ancora più esplicita: "[...]semita certe / tranquillae per virtutem patet unica vitae. / Nullum numen habes, si sit prudentia: nos te, / nos facimus, Fortuna, deam caeloque locamus" (363-66) ${ }^{27}$. La didascalia generale della seconda giornata prescrive di raccontare "di chi, da diverse cose infestato, sia oltre alla sua speranza, riuscito a lieto fine": ma la regina Filomena, precedentemente, aveva chiamato più esplicitamente in causa la fortuna (I Concl., 10). Se la novella di Alatiel mostra echi evidenti del testo di Giovenale, e se in esso la fortuna ha peso dominante, è lecito domandarsi se Boccaccio, secondo una sua tipica mossa,

26Per i due aspetti cfr. rispett. Velli (1995, p. 244-247) e Segre (1974).

27 Un sentiero solo si apre a una vita tranquilla: quello della virtù. Se regna la saggezza, non avrai altro nume. Noi, solo noi, Fortuna, ti rendiamo dea e ti collochiamo in cielo. 
non abbia creato per sporogenesi altre unità narrative originate da quel nucleo.

Approfittando dell'eccellente analisi di Velli a proposito del riuso giovenaliano nella novella di Alatiel, si può forse suggerire che una porzione del medesimo testo, o meglio un suo spunto, sia rintracciabile dietro la novella di Rinaldo d'Asti, cioè di un racconto, si badi, della medesima giornata (II 2). Si capisce che la base sia offerta dalla popolarità della leggenda di san Giuliano, oltre a tutto annotata da Boccaccio nel suo Zibaldone Magliabechiano, come ricorda Branca (BOCCACCIO, 1980, p. 141-142) nel suo commento; ma l'intrecciarsi di motivi differenti e di fonti anche tra loro non solo remote ma disparate (qui, dalle pratiche devozionali ai classici) è prassi in lui consueta. Si può sostenere che se il veicolo è offerto effettivamente da quella leggenda, il motore è forse da cercare altrove, dato che il tema della giornata è offerto dai rivolgimenti della fortuna, e dalla possibilità di ottenere insperati successi dove quella manchi. E in effetti Rinaldo, derubato dai tre masnadieri di denari, panni e cavallo, abbandonato dal suo servitore, morirebbe di freddo se non fosse riconfortato di tutto dalla bella vedova: bagno caldo, panni, cena e compagnia amorosa. Non solo; perché, all'atto di congedarlo, la donna gli "empie la borsa di denari", sicché al termine del racconto, riavuti, oltre al resto della refurtiva ("né ne perdé altro che un paio di cintolini"; II 2 41), i denari rubatigli, avrà anche aumentato il contante. Nella sua nota, Branca (BOCCACCIO, 1980, p. 151) rimarca "il carattere assolutamente straordinario (anche troppo)" degli avvenimenti. La straordinarietà potrebbe essere qui non deroga al verosimile decameroniano, ma originata dal fatto che alla base del racconto, più che un episodio non ordinario, c'è forse ancora il rovesciamento di una sentenza sempre dalla decima satira di Giovenale, con quel tanto di astrattamente eccessivo che si trasferisce dalla fonte al suo riuso. Parlando della vanità dei desideri di ricchezza, Giovenale scrive: "Nocte iter ingressus, gladium contumque timebis / et mota ad lunam trepidabis harundins umbra: / cantabit vacuus coram latrone viator" (X 20-22) ${ }^{28}$. Ebbene, Rinaldo proprio quegli avvisi trascura (porta con sé il contante, continua il viaggio anche a ora tarda, si accompagna a estranei), e ciò nonostante vince su tutti i fronti (anche economici). Non ci fosse la vicinanza della novella di Alatiel, e la sicurezza della sua derivazione giovenaliana, sarebbe certo necessaria maggiore cautela; ma quella contiguità suggerisce che i due racconti facciano in qualche modo sistema, costituendo un dittico sulla buona sorte ottenuta "oltre alla propria speranza". Così come a ogni tragica scomparsa dei suoi amanti si presenta per Alatiel una diversa avventura erotica, allo stesso modo tutte le perdite di Rinaldo si trasformano in nuovi e inaspettati guadagni. Anche se così fosse,

28 Se ti metti in viaggio di notte dovrai temere randelli e spade, e così morire di spavento per l'ombra di una canna che ondeggia al balenare della luna. Sotto il naso del ladro la canta chi viaggia a tasche vuote. 
come pare più che possibile, sarebbe in ogni caso difficile individuare la trafila genealogica che ha portato alla coppia di racconti esaminata. E ancora più difficile intendere se, una volta che dovesse essere accertata la fonte comune, si debba ritenere possibile una contemporaneità creativa tra i racconti; se cioè il disegno del libro cresca insieme con le novelle, o si determini in un momento successivo, all'atto dell'ordinamento del materiale ${ }^{29}$. Verosimile il fatto si sia prodotto quasi da sé (come conferma anche il processo di ribaltamento comune alle due novelle), una volta individuato il tema della giornata; e vi si aggiunga che i due protagonisti in pieno rispondono al cliché pertinente ai due sessi (il desiderio di guadagno per l'uomo, quello della bellezza per la donna; in realtà Giovenale prende di mira anche, e non meno, la bellezza maschile, con gli esempi di Ippolito e Bellerofonte). Ma in base a quale procedimento mentale la cosa sia avvenuta è arduo dire, perché affrontiamo i meccanismi della memoria di un autore che, come Boccaccio, ha confidenza piena coi suoi testi, con strumenti mentali inadeguati a descriverli. In altre parole, se Boccaccio condensa in pochi paragrafi della novella di Alatiel porzioni testuali consistenti della decima satira di Giovenale, per quale ragione, nel caso di quella di Rinaldo d'Asti, non agisce con altrettanta evidenza, potendosi ravvisare sì un riverbero, ma privo di agganci indiscutibili al testo? Una risposta potrebbe venire da quello che purtroppo ci manca, cioè dalla conoscenza del processo elaborativo del testo (ciò che invece ci è consentito nel caso del Canzoniere).

Logica vorrebbe che in tale frangente si dovesse ipotizzare una primogenitura legata al testo che accoglie in sé fatti verbali con certezza verificabili, anche se maneggiati con tale scaltrezza da essere passati inavvertiti per secoli (in questo caso, la novella di Alatiel), da cui poi si sarebbero staccati satelliti minori; il che spiegherebbe anche la scomparsa dei tratti testualmente più salienti in questi ultimi, avvenuta non tanto allo scopo di rendere meno palese la derivazione, ma per semplice innesto memoriale sul tronco della fonte prima. Con le parole di un maestro degli studi su Dante, altrettanto valide, in questa prospettiva, per Boccaccio, si può dire che, nell'avvicinarsi a quest'ultimo, la "Quellenforschung deve inevitabilmente rimanere elusiva almeno in parte: la sua era una mente per cui ogni assimilazione rappresentava istintivamente l'inizio di un'alchimia" (DRONKE, 1990, p. 14). Che i composti che l'alchimia producono siano per Boccaccio in parte differenti non cambia la sostanza della cosa.

Il discorso può trovare del resto ulteriore espansione in altra novella della seconda giornata, quella di Landolfo Rufolo, presentato come colui "al quale non bastando la sua ricchezza, disiderando di radoppiarla, venne presso che fatto di perder con tutta quella se stesso" (II 4 5). Dunque non tanto di desiderio di ricchezza, ma più specificamente di avidità si tratta in 
questo caso. La condanna dell'avidità è tratto talmente comune, dai pronunciamenti paolini (I Ad Tim., VI 9-10) alle esecrazioni dantesche e petrarchesche, che nulla sembrerebbe potersi dedurre. Né si tratta della prima volta che Boccaccio tocca il tema, affrontato già nel giovanile Filocolo (V 92 9), come ricorda Branca (BOCCACCIO, 1980, p. 167-168) nel suo commento. Un'esortazione a tenersi lontano dall'avidità, peraltro, Boccaccio la trova bell'e pronta, guarda caso, ancora nella stessa satira di Giovenale su citata. Ma, ribadisco, la trova per associazione spontanea della memoria, non per voluto ricorso a una sostanza libresca: "sed pluris nimia congesta pecunia cura / strangulat et cuncta exuperans patrimonia census / quanto delphinis Ballaena britannica maior" (12-14) [... $]^{30}$.

Più che possibile è perciò che anche in questo caso lo spunto iniziale sia il medesimo. Ci si troverebbe perciò in presenza nientemeno che di un trittico giovenaliano nell'ambito della stessa giornata. Si potrebbe dedurne che si sia prodotto nel caso in questione un effetto "a cascata", così che l'occultamento dei tratti individuabili nei due ultimi racconti non risponderebbe a una calcolata e preventiva strategia; esso sarebbe piuttosto l'esito di una piena confidenza col testo base, che offre da sé, una volta messo a fuoco il tema della giornata, i passaggi fondamentali tramite i quali verificare il peso della fortuna nelle vicende umane. In altre parole, la memoria testuale, accertata nel caso della novella di Alatiel, ha innescato la memoria associativa, all'origine di quelle di Rinaldo d'Asti e di Landolfo Rufolo.Eppure, a dimostrazione di quanto l'individuazione delle fonti, e ancor più l'analisi del loro trattamento, sia uno degli snodi davvero nevralgici non solo per capire il modo di lavorare di Boccaccio, ma per intendere l'ambizione intellettuale che informa il testo d'intrattenimento per eccellenza della letteratura non solo italiana, eppure, non mi so risolvere ad escludere, per la novella di Landolfo Rufolo, l'apporto di un altro testo. Nelle sue Vite dei filosofi, Diogene Laerzio registra, tra i dicta memorabilia di Antistene, quello secondo il quale, a elogio dell'autosufficienza e dello sprezzo di ogni possesso, chi viaggia dovrebbe portare con sé provvigioni che, galleggiando sull'acqua, potessero scampare con lui al naufragio (VI 6).

La vicinanza con la vicenda decameroniana, resa evidente proprio dal rovesciamento di situazione (l'avido Landolfo vorrebbe morire piuttosto che tornare a casa in miseria, e si salva aggrappandosi a una cassa galleggiante sull'acqua che cerca in ogni modo di allontanare da sé, prima di aggrapparvisi disperatamente), pare manifesta. Boccaccio non può avere conoscenza del testo greco delle Vite, per la sua ben nota inadeguatezza in materia, né avere accesso a una versione latina, dato che la prima completa in tale lingua, dovuta ad Ambrogio Traversari (la cosiddetta, appunto, versio Ambrosiana), è portata a termine nel 1433 (DORANDI, 2009, p.

30 Ma ancora di più ne soffoca il denaro ammassato con troppa avidità e un patrimonio superiore a tutti gli altri, quanto la balena britannica è più gigantesca di un delfino. 
222-228, part. p. 223-224). Né quella sentenza pare reperibile negli autori che sono abituale fonte di Boccaccio in tale repertorio (Cicerone, Seneca, Valerio Massimo, ecc.).

Ma non si può certo escludere che la conoscesse tramite un qualche florilegio, tardo-latino o medioevale, da lui compulsato; con l'avvertenza che il detto sopra citato è da alcuni attribuito ad Aristippo, potrebbero essere chiamati in causa, per fare solo due titoli (ma parecchi altri potrebbero rispondere all'appello), il Liber de vita et moribus philosophorum per lungo tempo attribuito a Walter Burley (che largamente si basa su Diogene Laerzio, infatti costantemente chiamato in causa nella compilazione) ${ }^{31} \mathrm{o}$ il Compendiloquium de vita et dictis illustrium philosophorum di Giovanni di Galles - un cui ms., il Riccardiano 1230, è in possesso di Boccaccio, che vi appone un unico notabile (CURSI; FIORILLA, 2013, p. 54, n. 7). Solo parzialmente rielaborato rispetto a Diogene Laerzio e arricchito di frange narrative, il detto di Antistene, attribuito ad Aristippo (lo stesso nome del personaggio che addottrina Gisippo e Tito: X 8 6), si legge infatti nel brano seguente del Liber di Burley, o meglio dello pseudo-Burley (si avverta come la sentenza finale sia preceduta da una tempesta che spaventa il filosofo, e da un naufragio che lo depone su un lido sconosciuto):

Navigante vero eo quandoque Corinthum et tempestate facta, nausiam passus est et expavit. Garrulus autem huic, tranquillitate reddita, dixit: "Quid est hoc quod nos quidem idiote intrepidi sumus, vos autem philosophi trepidatis?” Respondit: "Quia non de simili anima studemus. Te etenim pro nequissimi nebulonis anima nequaquam decuit esse solicitum, ego vere obnoxius debui de philosophi morte dolere, nam et homines divites amplius fures metuunt quam inopes". [...] Vitruvius vero in libro de architectura sic dicit: Aristippus, philosophus, Socratis discipulus, naufragio eiectus cum ad rodinense litus animadvertisset geometrica scemata descripta exclamavit ad comites: "Non desperemus, hominum quidem vestigia ego video". Statimque in oppidum rodinense conscendit et in gimnasium devenit ibique de philosophia disputans tantis muneribus ditatus est ut non tantum se ornaret, sed eciam eis qui cum eo erant et vestitum et cetera que opus essent ad victum praestaret.

Cum autem eius comites reverti in patriam voluissent interrogarentque eum quid vellet domi renunciari, ita mandavit dici atheniensibus: eas possesiones et viatica liberis oportere parari que eciam e naufragio una possent enatare cum salvatis, et ea vera vite

31 Per Diogene Laerzio quale fonte (insieme ad altri autori) di Burley, cfr. Knust (1964, p. 400-405) e Dorandi (2009, p. 201-212). La segnalazione di Burley come fonte filosofica boccacciana in Padoan (BOCCACCIO, 1965, p. 783 n. 103 , p. 843 n. 380 e 382). L'attribuzione del testo a un anonimo autore settentrionale della prima metà del Trecento è sostenuta da Grignaschi (1990); qualche cautela al riguardo in Petoletti (2000, p. 35-36). 
Si capisce che non tutto scorra con perfetto parallelismo nella riproposizione parodicamente rovesciata dei detti di Aristippo. Ma tanto la peculiarità della raccomandazione finale, situata perciò in zona facilmente memorizzabile ("eas possesiones [...] possent enatare"; non a ognuno, come al mercante boccacciano, capita che i propri averi stiano effettivamente a galla con sé sull'acqua), quanto la messa in guardia dalle mutazioni di fortuna e dalla perdita dei beni privati, sono segnali da considerare, dato che la cassa che salva la vita a Landolfo è carica di gioie che raddoppiano la ricchezza iniziale del protagonista. Ciò che accomuna la storia di quest'ultimo con molti dei casi sin qui esaminati è il trattamento, asseverativo (VI 9) o antifrastico (II 7), della sentenza desumibile dal repertorio della classicità, sminuzzata in segmenti dossografici o apoftegmatici e situata al punto di svolta del racconto.

Quanto detto sin qui non deve stupire, se si pensa al valore centrale che, nella decima satira di Giovenale, assume la riflessione sulla fortuna; fortuna che della seconda giornata decameroniana è esclusivo oggetto. Piuttosto, ci si deve interrogare sulla direzione intrapresa dalla riscrittura di Boccaccio, il quale, nei frangenti messi sotto osservazione, sottopone a scrutinio critico, e talora esplicitamente smentisce, quanto dichiara la sua fonte. Di Alatiel, sulla scorta dell'analisi di Velli, si è detto. Ma il discorso è da condurre con maggior delicatezza per le altre due novelle analizzate. Si potrà dire, in questo caso, che Boccaccio non intenda in alcun modo sottovalutare il ruolo della fortuna, cosa che il Decameron a ogni piè sospinto insegna (con massima evidenza nella programmatica premessa di Pampinea all'interno di questa stessa giornata; II 3 4-5); ma che, allo stesso tempo, metta in guardia contro ogni atteggiamento fatalistico nei confronti di quella. Proprio in ragione della sua imprevedibilità, non necessariamente si deve considerarne esclusivamente il volto arcigno, dato che ogni rovescio può convertirsi nel suo contrario. Tutto sta a trarne la lezione che a ognuno spetta; infatti, Landolfo Rufolo, prototipo, con Salabaetto, del mercante decameroniano deluso dalla sua attività (avrà ancora senso parlare dell'epopea dei mercatanti?), rinuncia a esercitare in futuro la mercatura, e a tentare ulteriormente la sorte. Se

32 Mentre navigava verso Corinto si alzò una tempesta, fu preso dalla nausea ed ebbe paura. Tornata la calma, un chiacchierone gli disse: "Com'è questo fatto, che noi persone comuni rimaniamo impavidi, e voi filosofi avete paura?" Gli rispose: "Perché non ci occupiamo dello stesso tipo di anima. Non ti è stato in alcun modo necessario preoccuparti per l'anima di un tipaccio da nulla, io invece ho dovuto dolermi per la morte di un filosofo, così come i ricchi temono i ladri più dei poveri". [...] Vitruvio nel libro De architectura scrive quanto segue: Aristippo, filosofo, discepolo di Socrate, sbalzato dal naufragio sul lido di Rodi, avendo notato sulla sabbia delle figurazioni in forma geometrica disse ai compagni: "Non disperiamo, vedo sicure tracce umane". Salì subito verso la città di Rodi e si recò al ginnasio; qui, disputando di filosofia, si arricchì con doni così numerosi che poté non solo vestire adeguatamente se stesso, ma anche provvedere di vestiti e altre cose necessarie al vitto i suoi compagni. Questi ultimi, volendo tornare in patria, e interrogandolo sul perché volesse rinunciare al ritorno, comandò che fosse detto questo agli ateniesi: che per gli uomini liberi è necessario avere a disposizione quei beni e quelle provviste di viaggio che possono scampare al naufragio insieme a chi sopravvive; e che dovevano ritenere vere difese della vita quelle alle quali né la tempesta iniqua della fortuna, né la mutazione delle condizioni politiche, né il saccheggio delle ricchezze private possono mai nuocere. 
quanto propongo in relazione a questa costellazione di racconti dovesse essere fondato, se ne potrebbe dedurre la validità di quanto anticipato sopra: la necessità di muovere dal particolare per cogliere il senso dell'operazione nel suo insieme.

Il fatto è che al senso dell'operazione, soprattutto in relazione al trattamento delle fonti, ci accostiamo ancora con inerzia mentale, avendo di mira soprattutto il cosa, mentre la combinazione vincente dovrebbe sempre accostarvi il come (attesa l'elaborazione su più livelli sopra ricordata). Per chiarire il punto, aggiungo un ultimo caso, la novella di Pietro Boccamazza e l'Agnolella (V 3). Il racconto, piuttosto che a narrazioni greco-bizantine, pare assimilabile ai romanzi cavallereschi medioevali. Si possono però indicare altre e più sottili commessure. La quinta giornata riscrive positivamente molte narrazioni infauste della tornata precedente, in particolare il racconto iniziale, terminante colla sepoltura congiunta dei due amanti (conclusione comune anche ad altri episodi di quella giornata: $7,8,9$ ). Il rito, più che al sigillo della narrazione di Tristano e Isotta (come spesso si legge), rimanda a quello della vicenda di Piramo e Tisbe, cui, per il particolare, anche la leggenda medioevale va apparentata ${ }^{33}$. Ma Boccaccio procede a una sistematica opera di ferializzazione del mitologico e del sacro, in aggiornamento continuo delle esperienze classiche, e in riscrittura versipelle di episodi scritturali e patristici, o della letteratura monastica ${ }^{34}$. Pietro e l'Agnolella sono sì eroi storicamente plausibili della Roma primotrecentesca, e la conclusione della loro vicenda è fausta, ma alcuni particolari del racconto hanno come velato ma presente sottofondo la storia ovidiana. L'opposizione della famiglia di Pietro e la sortita notturna sono fatti topici, ma altri dettagli assumono rilevanza diversa. Si leggano in successione i seguenti passi:

La giovane, veggendo che l'ora era tarda, ancora che le parole del vecchio [il padrone della casetta presso cui si rifugia l'Agnolella] la spaventassero, disse: "Se a Dio piacerà, Egli ci guarderà e voi e me di questa noia [essere assaliti dai briganti, con "dispiacere e vergogna" per la ragazza]; la quale se pur m'avenisse, è molto men male esser dagli uomini straziata che sbranata per li boschi dalle fiere" (29).

[...] avvenne che uno di loro [i briganti che entrano in casa della coppia di anziani che protegge l'Agnolella], non sappiendo altro che farsi, gittò la sua lancia nel fieno e assai vicin fu a uccidere la nascosa giovane e ella a palesarsi, per ciò che la lancia le venne allato alla sinistra poppa, tanto che col ferro le stracciò de' vestimenti, laonde ella fu per mettere un grande strido temendo d'esser fedita; ma

33 Per la presenza pervasiva della vicenda ovidiana nell'opera di Boccaccio, cfr. Filosa (2012, p. 92-98, 109-111) 34 Per questi ultimi aspetti, cfr. almeno Delcorno (1995) e il recente Paciucci (2010). 
ricordandosi là dove era, tutta riscossasi, stette cheta $(35$,$) .$

I due brani suggeriscono che qui sia all'opera una memoria dell'episodio dei due amanti babilonesi. Ma è la memoria del lettore a essere in difficoltà, perché essa deve quanto possibile accostarsi a quella attiva nel procedimento dell'autore, che giuoca le proprie carte anche su segmenti che passano inosservati, mentre la prima (quella del lettore) tende a procedere per inerzia, affidandosi alle operazioni automatiche anziché a quelle controllate dalla riflessione sul dato specifico (nel caso, la sempre imprevedibilmente peculiare elaborazione boccacciana della fonte $)^{35}$. Tisbe, si sa, non viene sbranata dalla leonessa che si abbevera alla fonte dopo aver fatto strage di un armento (Ovidio, Met., IV 96-98), ed è precisamente l'equivoco in cui cade il sopraggiunto Piramo a innescare la tragedia dei due fanciulli; ma è questo, per l'appunto, e non per caso, il timore dell'Agnolella. Quanto al secondo passo, l'indugio specifico è frutto sì della lenticolare attenzione descrittiva di Boccaccio, ma si deve essere allertati proprio dalla gratuità del gesto del brigante, la cui funzione si percepisce appieno se si ricordano i versi ovidiani di poco successivi all' apparire della fiera: "ut lea saeva sitim multa conpescuit unda, / dum redit in silvas, inventos forte sine ipsa / ore cruentato tenues laniavit amictus" ${ }^{36}$ (ivi, 102-4). Una sutura tra i due lacerti mostra come l'episodio di Piramo e Tisbe resista come sfondo potenzialmente mortale alla vicenda invece fortunata di Pietro e dell' Agnolella, di cui la veste stracciata della giovane donna è una sorta di relitto in forma di metonimia, un presagio di morte non realizzatasi. Che la storia abbia esito felice è infatti un altro degli innumerevoli capovolgimenti su cui Boccaccio si esercita. D'altra parte, se si percepisce, e giustamente, un sottofondo dantesco nella figura della giovane che "si mise tanto fralla selva, che ella non poteva vedere il luogo donde in quella entrata era" (20: con passo che, oltre che a Purg., XXVIII 22-24, rimanda a Inf., XV 13-15), si deve fare lo stesso sforzo per individuare, nella complessa testura della novella, altri innesti; tenendo presente preliminarmente che essi non si presentano tutti con lo stesso livello di sbalzo, in secondo luogo che, come Dante è sottoposto a una massiccia pressione deallegorizzante, allo stesso modo Ovidio subisce una torsione che rende arduo individuarne la presenza, tanto più di fronte a una soluzione favorevole (analogamente a quanto accade per la novella di Bernabò e Zinevra).

35 Per il peso che il sistema intuitivo continua ad avere nei confronti del riflessivo, cfr. Kahneman (2011).

36 La leonessa feroce, placata a furia d'acqua la sete, mentre tornava al bosco, trovato per caso abbandonato a terra quel velo delicato, lo stracciò con le fauci sporche di sangue. 
Si assiste insomma una volta di più a quelle forme di collegamento rovesciato indagate da Forni, che illustra la prossimità di elementi figurali facenti capo a vicende di esito opposto nella fattispecie il discorso di Forni (2008, part. p. 33-37) tocca l'immagine del cuore strappato, che lega due vicende di esito antitetico, tragico nella novella di Ghismonda e comicamente felice in quella di Ricciardo e Caterina (IV 1 46, V 4 42). L'unica differenza, in tali esempi di gemmazione che mantengono inalterato l'ordito mentre oppongono il disegno della trama, è data dal fatto che negli ultimi due casi il racconto responsivo è interno al testo, e nel caso qui sotto osservazione esso è esterno; ma la logica è identica, e consiste nel primo frangente nel passaggio dal letterale al metaforico (il cuore è effettivamente strappato a Guiscardo, mentre si presenta come fantasma di morte, comicamente scampata, a Ricciardo), e nel secondo nell'assunzione di una serie di immagini che trascorrono da un testo all'altro, consentendo, tramite l'inversione di rotta di svolte decisive del racconto, una conclusione positiva. La domanda da farsi è allora la seguente: per individuare la fonte di un autore smaliziato come Boccaccio è sufficiente cercare gli antecedenti manifesti delle singole narrazioni? O non occorre, come non solo in questo caso accade, avere attenzione anche a innesti specifici, sui quali di fatto si avvolge il resto del racconto? La risposta è ovvia, ma la strada da farsi è solo agli inizi.

$\mathrm{Si}$ capisce che quelli attraversati non siano che esempi, atti a illustrare alcuni utilizzi dei propri modelli da parte di Boccaccio, al fine di intraprendere strade narrative nuove. Essi comunque confermano quanto ancora occorra lavorare per intendere la ricchezza e la complessità sottostanti al progetto del Decameron. La veste problematica della narrazione boccacciana avvalora la giustezza del pronunciamento di Benjamin (1962, p. 241-242, 251), per il quale romanzo e racconto si contrappongono in quanto al primo pertiene il "senso della vita" e al secondo la "morale della storia" in un esercizio di ininterrotta interpretazione; ciò che trova nel Decameron la sua più radicale possibilità di verifica. In ogni caso, non c'inganni l'ampiezza dell'entrare. Dato il carattere elusivo della raccolta, la facilità d'accesso, che può celare trabocchetti culturalmente insidiosi, è in funzione dell'uso responsabile che del libro ogni lettore deve saper fare. 


\section{Riferimenti bibliografici}

BARBI, M. Sul testo del 'Decameron'. In La nuova filologia e l'edizione dei nostri scrittori, da Dante al Manzoni. Firenze: Sansoni, 1973.

BATTAGLIA RICCI, L. Ragionare nel giardino. Boccaccio e i cicli pittorici del «Trionfo della morte». Roma: Salerno 1987.

BATTAGLIA RICCI, L. Boccaccio. Roma: Salerno, 2000.

BATTAGLIA RICCI, L. Edizioni d'autore, copie di lavoro, interventi di autoesegesi: testimonianze trecentesche. In "Di mano propria". Gli autografi dei letterati italiani, Atti del Convegno Internazionale di Forlì, 24-27 novembre 2008, a cura di Guido Baldassarri, Matteo Motolese, Paolo Procaccioli, Emilio Russo. Roma: Salerno, 2010. p. 123-157.

BATTAGLIA RICCI, L. Scrivere un libro di novelle. G. Boccaccio autore, lettore, editore. Ravenna: Longo, 2013.

BENJAMIN, W. Il narratore. Considerazioni sull'opera di N. Leskov. In Angelus Novus. Saggi

e frammenti. Traduzione e introduzione di Renato Solmi. Torino: Einaudi, 1962. p. 235-260.

BERLIN, I. Il riccio e la volpe e altri saggi. A cura di Henry Hardy e Aileen Kelly, Introduzione di Aileen Kelly. Milano: Adelphi, 1998

BERNARDO DI CHIARAVALLE, SANTO. Patrologia latina, a cura di J.-P. Migne, vol. 183. Parigi: J.-P. Migne, 1844-1855.

BETTINZOLI, A. Per una definizione delle presenze dantesche nel 'Decameron'. I. I registri ‘ideologici’, lirici, drammatici. Studi sul Boccaccio, v. XIII, 1981-1982, p. 267-326.

BETTINZOLI, A. Per una definizione delle presenze dantesche nel 'Decameron'. II. Ironizzazione e espressivismo antifrastico-deformatorio. Studi sul Boccaccio, v. XIV, 1983-1984, p. 209-240.

BOCCACCIO, G. Decameron. A cura di Maurizio Fiorilla, illustrazioni di Mimmo Paladino. Roma: Istituto della Enciclopedia Italiana, 2011.

BOCCACCIO, G. Decameron. A cura di Vittore Branca. Torino: Einaudi, 1980.

BOCCACCIO, G. Decamerón. Edición de María Hernández Esteban, Traducción de María Hernández Esteban. Madrid: Catedra, 1994.

BOCCACCIO, G. Decameron. Introduzione, note e repertorio di Cose (e parole) del mondo di Amedeo Quondam, Testo critico e Nota al teso a cura di Maurizio Fiorilla, Schede introduttive e notizia biografica di Giancarlo Alfano. Milano: BUR Classici, 2013.

BOCCACCIO, G. Elegia di madonna Fiammetta. A cura di Carlo Delcorno. In BRANCA, V. (a cura di). Tutte le opere di G. Boccaccio. Milano: Mondadori, 1994. v. V, t. II, p. 3-412. 
BOCCACCIO, G. Esposizioni sopra la Comedia di Dante. A cura di Giorgio Padoan. In BRANCA, V. (a cura di). Tutte le opere di G. Boccaccio. Milano: Mondadori, 1965. v. VI.

BRAGANTINI, R. Ingressi laterali al Trecento maggiore. Dante, Petrarca, Boccaccio. Napoli: Liguori, 2012.

BRAGANTINI, R. La spola del racconto: dal proverbio alla novella, e viceversa. In Il proverbio nella letteratura italiana dal XV al XVII secolo, Atti delle giornate di studi, 5-6 dicembre 2012, Università Roma 3-Fondazione Marco Besso. Manziana (Roma): Vecchiarelli, 2014.

BRESCHI, G. Il ms. Parigino it. 482 e le vicissitudini editoriali del 'Decameron'. Postilla per A. Rossi, Medioevo e Rinascimento, XVIII/n.s. XV, 2004.

CHERCHI, P. L'onestade e l'onesto raccontare del 'Decameron'. Fiesole: Cadmo, 2004.

CONTE, A. (a cura di). Il Novellino. Presentazione di Cesare Segre. Roma: Salerno, 2001.

CONTINI, G. Dante come personaggio-poeta della 'Commedia'. In . Varianti e altra linguistica. Una raccolta di saggi (1938-1968). Torino: Einaudi, 1970. p. 335-361.

COSTANTINI, A. M. Studi sullo Zibaldone Magliabechino. II. Il florilegio senechiano. Studi sul Boccaccio, v. VIII, 1974, p. 79-126.

CURSI, M. Il 'Decameron': scritture, scriventi, lettori. Storia di un testo. Roma: Viella, 2007.

CURSI, M. La scrittura e i libri di G. Boccaccio. Roma: Viella, 2013.

CURSI, M.; FIORILLA, M. G. Boccaccio. In BRUNETTI, G., FIORILLA, M., PETOLETTI, M. (a cura di). Autografi dei letterati italiani. Le Origini e il Trecento. Roma: Salerno, 2013, t. I, p. 43-103.

DELCORNO, C. Note sui dantismi nell' 'Elegia di Madonna Fiammetta'. In Studi sul Boccaccio, v. XI, 1979, p. 251-294.

DELCORNO, C. Ironia/parodia. In BRAGANTINI, R.; FORNI, P. M. (a cura di). Lessico critico decameroniano. Torino: Bollati Boringhieri, 1995. p. 162-191.

DORANDI, T. Laertiana. Capitoli sulla tradizione manoscritta e sulla storia del testo delle 'Vite dei filosofi' di Diogene Laerzio. Berlin; NewYork: de Gruyter, 2009.

DRONKE, P. Dante e le tradizioni latine medievali. Bologna: il Mulino, 1990.

FILOSA, E. Tre studi sul De mulieribus claris. Milano: LED, 2012.

FIORILLA, M. Per il testo del ‘Decameron'. In L’Ellisse, V, 2010, p. 9-38.

FIORILLA, M. Ancora per il testo del 'Decameron'. In L'Ellisse, VIII, 2013, 1, p. 75-90.

FORNI, P. M. Forme complesse nel Decameron. Firenze: Olschki, 1992.

FORNI, P. M. Parole come fatti. La metafora realizzata e altre glosse al 'Decameron'. Napoli: Liguori, 2008. 
FUMAGALLI, E. Boccaccio e Dante. In ROBERTIS, T. et al. (a cura di). Boccaccio autore e copista. Firenze: Mandragora, 2013. p. 25-31 (Catalogo della mostra Boccaccio autore e copista, Firenze, Biblioteca Medicea Laurenziana, 11 ottobre 2013-11 gennaio 2014).

GRIGNASCHI, M. Lo pseudo Walter Burley e il 'Liber de vita et moribus philosophorum'. In Medioevo, 16, 1990, p. 131-190.

HOLLANDER, R. Boccaccio's Dante and the Shaping Force of Satire. Ann Arbor: University of Michigan Press, 1997.

KAHNEMAN, D. Thinking, Fast and Slow. New York: Farrar, Straus and Giroux, 2011.

KNUST, H. (a cura di). Gualteri Burlaei Liber de vita et moribus philosophorum, mit einer altspanischen Übersetzung der Eskurialbibliothek. Tübingen: Bibliothek des Litterarischen Vereins in Stuttgart, 1886; Frankfurt a/Main: Minerva, 1964, Unveränderter Nachdruck.

MALAGNINI, F. Il sistema delle maiuscole nell'autografo berlinese del 'Decameron' e la scansione del mondo commentato. Studi sul Boccaccio, v. XXXI, 2003, p. 31-69.

NOCITA, T. Per una nuova paragrafatura del testo del 'Decameron'. Appunti sulle maiuscole del cod. Hamilton 90 (Berlin, Staatsbibliothek Preussischer Kulturbesitz). Critica del testo, II/3, 1999, p. 925-934.

PACIUCCI, M. Elementi della rielaborazione del sacro in Boccaccio. La novella VIII 7: Elena e lo scolare. In "Tout est dit". Teoria, problemi, fenomeni della riscrittura, Atti del Seminario di studi del Dottorato in Italianistica e Spettacolo, Roma 17-18 giugno 2009, a cura di Renzo Bragantini, Studi (e testi) italiani, 26 (2010), p. 79-96.

PADOAN, G. Sulla genesi e la pubblicazione del 'Decameròn'. In .Il Boccaccio, le Muse, il Parnaso e l'Arno. Firenze: Olschki, 1978, p. 93-121.

PETOLETTI, M. Il 'Chronicon' di Benzo d'Alessandria e i classici latini all'inizio del XIV secolo. Edizione critica del libro XXIV: 'De moribus et vita philosophorum'. Milano: Vita e Pensiero, 2000.

PETRARCA, F. Canzoniere. A cura di Marco Santagata. Edizione commentata, nuova edizione aggiornata. Milano: Mondadori, 2004.

PETRARCA, F. Il codice degli abbozzi: edizione e storia del manoscritto Vaticano latino 3196. A cura di Laura Paolino. Milano; Napoli: Ricciardi, 2000.

PETRARCA, F. Le familiari. Traduzione e cura di Ugo Dotti, testo critico di Vittorio Rossi e Umberto Bosco, collaborazione di Felicita Audisio, 5 t. Torino: Aragno, 2004-2009.

PETRARCA, F. Le "Senili" secondo l'edizione Basilea 1581. A cura di Marziano Guglielminetti, con un contributo critico di Mario Martelli e una nota di Patrizia Pellizzari. Savigliano (CU): L'Artistica, 2006.

PETRUCCI, A. Il libro manoscritto. In Letteratura italiana, diretta da Alberto Asor Rosa, v. II, Produzione e consumo. Torino: Einaudi, 1983, p. 497-524. 
PICONE, M. L'invenzione della novella italiana. Tradizione e innovazione. In La novella italiana, Atti del Convegno di Caprarola, 19-24 settembre 1988, 2 t. Roma: Salerno, 1989, I, p. 119-154.

POZZI, G. Petrarca, i Padri e soprattutto la Bibbia. In Alternatim. Milano: Adelphi, 1996, p. 143-189.

RAFTI, P. "Lumina dictionum". Interpunzione e prosa in G. Boccaccio. IV. Studi sul Boccaccio, v. XXIX, 2001, p. 3-66.

SANTAGATA, M. I frammenti dell'anima. Storia e racconto nel Canzoniere di Petrarca. Bologna: il Mulino, 2004.

SEGRE, C. Comicità strutturale nella novella di Alatiel. In Le strutture e il tempo. Torino: Einaudi, 1974, p. 145-159.

VELLI, G. Seneca nel 'Decameron'. Giornale storico della letteratura italiana, CLXVIII, 1991, p. 321-334.

VELLI, G. Memoria. In BRAGANTINI, R.; FORNI, P. M. (a cura di ). Lessico critico decameroniano. Torino: Bollati Boringhieri, 1995, p. 222-248.

VITALE, M.; BRANCA, V. Il capolavoro del Boccaccio e due diverse redazioni, 2 t.; I: Vitale, Maurizio, La riscrittura del 'Decameron'. I mutamenti linguistici; II: Branca, Vittore, Variazioni narrative e stilistiche. Venezia: Istituto Veneto di Scienze, Lettere ed Arti, 2002. 